\title{
Fourth Order Compact Finite Difference Method for Solving Singularly Perturbed 1D Reaction Diffusion Equations with Dirichlet Boundary Conditions
}

\author{
Fasika Wondimu ${ }^{1}$, Gemechis File ${ }^{2 *}$ and Tesfaye Aga $^{2}$ \\ ${ }^{1}$ Department of Mathematics, Dilla University, Dilla, Ethiopia \\ ${ }^{2}$ Department of Mathematics, Jimma University, Jimma, Ethiopia (*gammeef@yahoo.com)
}

\begin{abstract}
A numerical method based on finite difference scheme with uniform mesh is presented for solving singularly perturbed two-point boundary value problems of 1D reaction-diffusion equations. First, the derivatives of the given differential equation is replaced by the finite difference approximations and then, solved by using fourth order compact finite difference method by taking uniform mesh. To demonstrate the efficiency of the method, numerical illustrations have been given. Graphs are also depicted in support of the numerical results. Both the theoretical and computational rate of convergence of the method have been examined and found to be in agreement. As it can be observed from the numerical results presented in tables and graphs, the present method approximates the exact solution very well.
\end{abstract}

Keywords: Singular perturbation, Compact finite difference method, Reaction diffusion.

\section{INTRODUCTION}

Any differential equation in which the highest order derivative is multiplied by a small positive parameter $\varepsilon(0<\varepsilon<<1)$ is called Singular Perturbation Problem and the parameter is known as the perturbation parameter. These types of problems arise very frequently in diversified fields of applied mathematics and engineering, for instance fluid mechanics, elasticity, hydrodynamics, quantum mechanics, chemical-reactor theory, aerodynamics, plasma dynamics, rarefied-gas dynamics, oceanography, meteorology, modeling of semiconductor devices, diffraction theory and reaction-diffusion processes and many other allied areas.

It is well-known fact that the solution of singular perturbation problems exhibits a multi-scale character; that is, there are thin transition layer(s) where the solution varies rapidly or jump suddenly known as boundary layer, while away from the layer(s) the solution behaves regularly and varies slowly known as outer region. Thus, the treatment of such problems is not trivial because of the boundary layer behavior of their solutions. Detailed theory and analytical discussions of solving singular perturbation problems have been published (O'Malley, 1974, 1991; Nayfeh, 1973, 1981; Cole and Kevorkian, 1979; Bender and Orszag, 1978; Eckhaus, 1973; Vandyke, 1975; Bellman, 1964), and have the details of numerical and asymptotic solutions in 
(Doolan et al., 1980; Goering et al., 1983; Hemker, 1977; Hemker and Miller, 1979; Miller, 1993; Miller et al., 1996).

Moreover, in the recent times many researchers have been trying to develop and present numerical methods for solving these problems. For instance, Padmaja et al. (2012) have presented a nonstandard explicit method involving the reduction of order for solving singularly perturbed two point boundary value problems. To apply the method, the authors have approximated the original problem by a pair of initial value problems and solved the first initial value problem as outer region problem whose solution can be required in the second initial value problem which they considered it as an inner region problem and is modified using the stretching transformation. The Differential Quadrature Method (DQM) has been applied for finding the numerical solution of singularly perturbed two point boundary value problems with mixed condition (Prasad and Reddy, 2011). DQM is based on the approximation of the derivatives of the unknown functions involved in the differential equations at the mesh point of the solution domain and is an efficient discretization technique in solving boundary value problems using a considerably small number of grid points. Geng (2011) has proposed the reproducing kernel method (RKM) for solving a class of singularly perturbed boundary value problems by transforming the original problem in to a new boundary value problem whose solution does not change rapidly. RKM has the advantage that it can produce smooth approximate solutions, but it is difficult to apply the method for singularly perturbed boundary value problems without transforming using appropriate transformation. However, most of the existing classical finite difference methods which have been used in solving singular perturbation problems give good result only when the mesh size is much less than the perturbation parameter which is very costly and time consuming.

In this paper, fourth order compact finite difference method is presented for solving singularly perturbed 1D reaction-diffusion equations. Compact finite difference method is a finite difference method which employs a linear combination of three consecutive points of derivatives to approximate a linear combination of the same three consecutive values of a function $y\left(x_{j}\right), \quad j=i-1, i, i+1$. 


\section{DESCRIPTION OF THE METHOD}

Consider the following singularly perturbed reaction-diffusion equation of the form:

$$
-\varepsilon y^{\prime \prime}(x)+a(x) y(x)=f(x) ; \quad 0 \leq x \leq 1,
$$

with the boundary conditions

$$
y(0)=\alpha, y(1)=\beta
$$

where, $\varepsilon$ is a small positive parameter (diffusion coefficient) such that $0<\varepsilon<<1, \alpha, \beta$ are given constants and $a(x) ; f(x)$ are assumed to be sufficiently continuously differentiable functions. Furthermore, assume that $a(x) \geq \gamma>0$ in the interval $[0,1]$, where $\gamma$ is some positive constant. To describe the method, we divide the interval $[0,1]$ into $N$ equal sub-intervals of mesh length $h$. Let $0=x_{0}, x_{1}, x_{2}, \ldots, x_{N}=1$ be the mesh points. Then, we have $x_{i}=x_{0}+i h, i=0,1,2, \ldots, N$ For the sake of simplicity, let us denote $a\left(x_{i}\right)=a_{i}, \quad f\left(x_{i}\right)=f_{i}, y^{\prime}\left(x_{i}\right)=y_{i}^{\prime}, \quad y^{\prime \prime}\left(x_{i}\right)=y_{i}^{\prime \prime}$ and $y^{(n)}\left(x_{i}\right)=y_{i}^{(n)}$. Assume that $y(x)$ has continuous fourth order derivatives on $[0,1]$.

By using Taylor series expansion, we obtain:

$$
\begin{aligned}
& y_{i+1}=y_{i}+h y_{i}^{\prime}+\frac{h^{2}}{2 !} y_{i}^{\prime \prime}+\frac{h^{3}}{3 !} y_{i}^{(3)}+\frac{h^{4}}{4 !} y_{i}^{(4)}+\frac{h^{5}}{5 !} y_{i}^{(5)}+\frac{h^{6}}{6 !} y_{i}^{(6)}+O\left(h^{7}\right) \\
& y_{i-1}=y_{i}-h y_{i}^{\prime}+\frac{h^{2}}{2 !} y_{i}^{\prime \prime}-\frac{h^{3}}{3 !} y_{i}^{(3)}+\frac{h^{4}}{4 !} y_{i}^{(4)}-\frac{h^{5}}{5 !} y_{i}^{(5)}+\frac{h^{6}}{6 !} y_{i}^{(6)}+O\left(h^{7}\right)
\end{aligned}
$$

Subtracting Eq. (4) from Eq. (3), we obtain the second order finite difference approximation $\left(\delta_{c}^{1} y_{i}\right)$ for the first derivative of $y_{i}$ :

$$
\delta_{c}^{1} y_{i}=\frac{y_{i+1}-y_{i-1}}{2 h}+\tau_{1}
$$

where, $\tau_{1}=-\frac{h^{2}}{6} y_{i}^{(3)}$.

Similarly, adding Eqs. (3) and (4), we obtain the second order finite difference approximation $\left(\delta_{c}^{2} y_{i}\right)$ for the second derivative of $y_{i}$ :

$$
\delta_{c}^{2} y_{i}=\frac{y_{i+1}-2 y_{i}+y_{i-1}}{h^{2}}+\tau_{2}
$$

where, $\tau_{2}=-\frac{h^{2}}{12} y_{i}^{(4)}$.

CNCS, Mekelle University 
Substituting Eqs. (3)and(4) into Eq. (5), we obtain:

$$
\delta_{c}^{1} y_{i}=y_{i}^{\prime}+\frac{h^{2}}{6} y_{i}^{(3)}+\tau_{3}
$$

where, $\tau_{3}=\frac{h^{4}}{120} y_{i}^{(5)}+\tau_{1}$.

Substituting Eqs. (3) and (4) into Eq. (6), we obtain: $\delta_{c}^{2} y_{i}=y_{i}^{\prime \prime}+\frac{h^{2}}{12} y_{i}^{(4)}+\tau_{4}$

where, $\tau_{4}=\frac{h^{4}}{360} y_{i}^{(6)}+\tau_{2}$.

Writing Eq. (1) at discretized mesh, we obtain: $-\varepsilon y_{i}^{\prime \prime}+a_{i} y_{i}=f_{i}$

Differentiating Eq. (9) twice and solving for $y_{i}^{(4)}$, we get: $y_{i}^{(4)}=\frac{a_{i}}{\varepsilon} y_{i}^{\prime \prime}-\frac{f_{i}^{\prime \prime}}{\varepsilon}$

Substituting Eq. (10) into Eq. (8) and solving for $y_{i}^{\prime \prime}$ is given by:

$$
y_{i}^{\prime \prime}=\frac{\delta_{c}^{2} y_{i}+\frac{h^{2}}{12 \varepsilon} f_{i}^{\prime \prime}-\tau_{4}}{1+\frac{a_{i} h^{2}}{12 \varepsilon}}
$$

Substituting Eq. (11) into Eq. (1) for $y_{i}^{\prime \prime}$ yields:

$$
-\varepsilon \delta_{c}^{2} y_{i}-\frac{h^{2}}{12} f_{i}^{\prime \prime}+\varepsilon \tau_{4}+a_{i} y_{i}\left(1+\frac{a_{i} h^{2}}{12 \varepsilon}\right)=f_{i}\left(1+\frac{a_{i} h^{2}}{12 \varepsilon}\right)
$$

Substituting Eq. (6) for $\delta_{c}^{2} y_{i}$ into Eq. (12), we obtain:

$$
-\frac{\varepsilon}{h^{2}} y_{i-1}+\left(\frac{2 \varepsilon}{h^{2}}+a_{i}+\frac{a_{i}{ }^{2} h^{2}}{12 \varepsilon}\right) y_{i}-\frac{\varepsilon}{h^{2}} y_{i+1}=\left(1+\frac{a_{i} h^{2}}{12 \varepsilon}\right) f_{i}+\frac{h^{2}}{12} f_{i}^{\prime \prime}+\tau
$$

where, $\tau=-\frac{\varepsilon h^{4}}{360} y_{i}^{(6)}$ is the local truncation error of the method.

From Eq. (13) we get three-term recurrence relation of the form:

$$
-E_{i} y_{i-1}+F_{i} y_{i}-G_{i} y_{i+1}=H_{i}, \quad i=1,2,3, \ldots, N-1
$$

where, $E_{i}=\frac{\varepsilon}{h^{2}}, F_{i}=\frac{2 \varepsilon}{h^{2}}+a_{i}+\frac{a_{i}{ }^{2} h^{2}}{12 \varepsilon}, G_{i}=\frac{\varepsilon}{h^{2}}, H_{i}=\left(1+\frac{a_{i} h^{2}}{12 \varepsilon}\right) f_{i}+\frac{h^{2}}{12} f_{i}^{\prime \prime}$ 
Eq. (14) can easily be solved by Discrete Invariant Imbedding Algorithm commonly known as Thomas Algorithm. To get the numerical results and validate the scheme, MATLAB software has been applied.

The conditions for the discrete invariant imbedding algorithm to be stable are, (see Angel and Bellman, 1972; Elsgolt's and Norkin, 1973):

$$
E_{i}>0, G_{i}>0, \quad F_{i} \geq E_{i}+G_{i} \text { and }\left|E_{i}\right| \leq\left|G_{i}\right|
$$

For our method, one can easily show that Eq. (14) satisfies the conditions given above and hence Thomas Algorithm is stable for the proposed method.

\section{CONVERGENCE ANALYSIS}

Writing the tri-diagonal system in Eq. (14) above in matrix vector form, we get:

$$
A Y=C
$$

where, $A=\left(m_{i j}\right), 1 \leq i, j \leq N-1$ is a tri-diagonal matrix of order $N$, with

$$
\begin{aligned}
& m_{i i+1}=-\frac{\varepsilon}{h^{2}} \\
& m_{i i}=\frac{2 \varepsilon}{h^{2}}+a_{i}+\frac{a_{i}{ }^{2} h^{2}}{12 \varepsilon} \\
& m_{i i-1}=-\frac{\varepsilon}{h^{2}}
\end{aligned}
$$

and $C=\left(d_{i}\right)$ is a column vector with $d_{i}=\left(1+\frac{a_{i} h^{2}}{12 \varepsilon}\right) f_{i}+\frac{h^{2}}{12} f_{i}^{\prime \prime}, \quad$ for $i=1,2, \ldots, N-1$ with the local truncation error

$$
\tau_{i}(h)=-\frac{\varepsilon h^{4}}{360} y_{i}^{(4)}
$$

We also have $A \bar{Y}-\tau(h)=C$

where, $\bar{Y}=\left(\bar{y}_{0}, \bar{y}_{1}, \bar{y}_{2}, \ldots, \bar{y}_{N}\right)^{t}$ denote the exact solution and $\tau(h)=\left(\tau_{1}\left(h_{0}\right), \tau_{2}\left(h_{1}\right), \ldots, \tau_{N}\left(h_{N}\right)\right)^{t}$ denote the local truncation error. 
Making use of Eq. (15) and Eq. (17), we obtain an error equation:

$$
A E=\tau(h)
$$

where, $E=\bar{Y}-Y=\left(e_{0}, e_{1}, e_{2}, \ldots, e_{N}\right)^{t}$

Let $S_{i}$ be the sum of elements of the $i^{\text {th }}$ row of matrix $A$, then $S_{1}=\sum_{j=1}^{N-1} m_{1 j}$, for $i=1$.

Therefore, $S_{1}=\frac{\varepsilon}{h^{2}}+a_{i}+\frac{a_{i}{ }^{2} h^{2}}{12 \varepsilon}$, for $i=1$.

$$
\begin{aligned}
S_{i} & =\sum_{j=1}^{N-1} m_{i j}, \text { for } i=2,3, \ldots, N-2 \\
& =a_{i}+A_{0} h^{2}, \text { where } A_{0}=\frac{a_{i}^{2}}{12 \varepsilon}
\end{aligned}
$$

Therefore, $S_{i}=a_{i}+O\left(h^{2}\right)$, where $\left|a_{i}\right|=\min _{2 \leq i \leq N-2} S_{i}$, for $i=2,3, \ldots, N-2$.

$$
S_{N-1}=\sum_{j=1}^{N-1} m_{N-1, j} \text {, for } i=N-1 \text {, Therefore, } S_{N-1}=\frac{\varepsilon}{h^{2}}+a_{i}+\frac{a_{i}^{2} h^{2}}{12 \varepsilon} \text {, for } i=N-1 \text {. }
$$

Since, $0<\varepsilon<<1$, we can choose $h$ sufficiently small so that the matrix $A$ is irreducible and monotone (Mohanty and Jha, 2005). Then, it follows that $A^{-1}$ exists and its elements are nonnegative. Hence, from Eq. (18), we get

$$
E=A^{-1} \cdot \tau(h)
$$

and

$$
\|E\|=\left\|A^{-1}\right\| .\|\tau(h)\|
$$

Let $\bar{m}_{k, i}$ be the $(k, i)$ elements of $A^{-1}$. Since, $\bar{m}_{k, i} \geq 0$, by the definition of multiplication of matrices with its inverses, we have

$$
\sum_{i=1}^{N-1} \bar{m}_{k, i} S_{i}=1, \quad k=1,2, \ldots, N-1
$$

Therefore, it follows that $\quad \sum_{i=1}^{N-1} \bar{m}_{k, i} \leq \frac{1}{\min _{1 \leq i \leq N-1} S_{i}}=\frac{1}{\left|a_{i}\right|}$

We define $\left\|A^{-1}\right\|=\max _{1 \leq i \leq N-1} \sum_{i=1}^{N-1}\left|\bar{m}_{k, i}\right|$ and $\|\tau(h)\|=\max _{1 \leq i \leq N-1}\left|\tau_{i}\left(h_{i}\right)\right|$

CNCS, Mekelle University 
From Eqs. (16), (19), and (20) and (22), we obtain:

$$
\begin{aligned}
& e_{j}=\sum_{i=1}^{N-1} \bar{m}_{k, i} \cdot \tau_{i}(h), \text { for } j=1,2, \ldots, N-1 \\
& e_{j} \leq \frac{1}{\left|a_{i}\right|} \cdot \tau_{i}(h)
\end{aligned}
$$

Therefore, $e_{j} \leq \frac{k h^{4}}{\left|a_{i}\right|}$, for $j=1,2, \ldots, N-1$ where, $k=\left(\frac{\varepsilon}{360}\right)\left|y_{i}^{(6)}\right|$, which is a constant and independent of $h$. Therefore, $\|E\| \leq o\left(h^{4}\right)$. This implies that the method gives a fourth order convergence.

\section{NUMERICAL EXAMPLES}

To demonstrate the applicability of the methods, two model singularly perturbed problems have been considered. These examples have been chosen because they have been widely discussed in the literature and their exact solutions were available for comparison.

Example 1: Consider the following singularly perturbed problem:

$$
-\varepsilon y^{\prime \prime}+y=x, \quad 0 \leq x \leq 1, \text { with } y(0)=1, \quad y(1)=1+\exp \left(-\frac{1}{\sqrt{\varepsilon}}\right) .
$$

The exact solution is given by:

$$
y(x)=x+\exp \left(-\frac{x}{\sqrt{\varepsilon}}\right) .
$$

The numerical solutions in terms of maximum absolute errors are given in Tables 1 .

Example 2: Consider the following singularly perturbed problem:

$$
-\varepsilon y^{\prime \prime}+y=-\cos ^{2}(\pi x)-2 \varepsilon \pi^{2} \cos (2 \pi x), \quad 0 \leq x \leq 1, \text { with } y(0)=y(1)=0 .
$$

The exact solution for this example is given by:

$$
y(x)=\frac{\exp (-(1-x) / \sqrt{\varepsilon})+\exp (-x / \sqrt{\varepsilon})}{1+\exp (-1 / \sqrt{\varepsilon})}-\cos ^{2}(\pi x) .
$$

The numerical results of maximum absolute errors are tabulated in tables 2 for different values of the perturbation parameters $\varepsilon$ and $N$. The effect of the perturbation parameter on the solution of the problem is also shown in figures 1(a) - 2(b) for fixed $h$ and different values of the CNCS, Mekelle University 174

ISSN : 2220-184X 
perturbation parameter $\varepsilon$. That is, figures 1(a) - 2(b) show the comparison of the exact and numerical solutions for $h \geq \varepsilon$.

\section{NUMERICAL RESULTS}

Table 1. Maximum Absolute Errors for Example 1.

\begin{tabular}{|cccccc|}
\hline \multicolumn{2}{|c}{$N=16$} & $N=32$ & $N=64$ & $N=128$ & $N=256$ \\
\hline \multicolumn{7}{|c}{ Our Method } & & & \\
\hline $1 / 16$ & $1.9605 \mathrm{E}-006$ & $1.2339 \mathrm{E}-007$ & $7.7250 \mathrm{E}-009$ & $4.8297 \mathrm{E}-010$ & $2.9947 \mathrm{E}-011$ \\
\hline $1 / 32$ & $7.8196 \mathrm{E}-006$ & $4.9554 \mathrm{E}-007$ & $3.1124 \mathrm{E}-008$ & $1.9474 \mathrm{E}-009$ & $1.2164 \mathrm{E}-010$ \\
\hline $1 / 64$ & $3.0781 \mathrm{E}-005$ & $1.9774 \mathrm{E}-006$ & $1.2445 \mathrm{E}-007$ & $7.7918 \mathrm{E}-009$ & $4.8715 \mathrm{E}-010$ \\
\hline $1 / 128$ & $1.1257 \mathrm{E}-004$ & $7.8234 \mathrm{E}-006$ & $4.9578 \mathrm{E}-007$ & $3.1137 \mathrm{E}-008$ & $1.9482 \mathrm{E}-009$ \\
\hline \multicolumn{7}{|c|}{ Rashidinia et al. (2007) } & & & & \\
\hline $1 / 16$ & $2.96 \mathrm{E}-006$ & $1.85 \mathrm{E}-007$ & $1.15 \mathrm{E}-008$ & $7.24 \mathrm{E}-010$ & $4.56 \mathrm{E}-011$ \\
\hline $1 / 32$ & $1.18 \mathrm{E}-005$ & $7.54 \mathrm{E}-007$ & $4.67 \mathrm{E}-008$ & $2.96 \mathrm{E}-009$ & $1.82 \mathrm{E}-010$ \\
\hline $1 / 64$ & $4.74 \mathrm{E}-005$ & $2.96 \mathrm{E}-006$ & $1.86 \mathrm{E}-007$ & $1.16 \mathrm{E}-008$ & $7.30 \mathrm{E}-010$ \\
\hline $1 / 128$ & $1.78 \mathrm{E}-004$ & $1.18 \mathrm{E}-005$ & $7.46 \mathrm{E}-007$ & $4.67 \mathrm{E}-008$ & $2.92 \mathrm{E}-009$ \\
\hline
\end{tabular}

Table 2. Maximum Absolute Errors for Example 2.

\begin{tabular}{|cccccc|}
\hline \multicolumn{2}{|c}{$N=16$} & $N=32$ & $N=64$ & $N=128$ & $N=256$ \\
\hline Our Method & & & \\
\hline $1 / 16$ & $2.7142 \mathrm{E}-005$ & $1.6884 \mathrm{E}-006$ & $1.0540 \mathrm{E}-007$ & $6.5854 \mathrm{E}-009$ & $4.1160 \mathrm{E}-010$ \\
\hline $1 / 32$ & $1.3336 \mathrm{E}-005$ & $8.2769 \mathrm{E}-007$ & $5.1638 \mathrm{E}-008$ & $3.2259 \mathrm{E}-009$ & $2.0159 \mathrm{E}-010$ \\
\hline $1 / 64$ & $3.5441 \mathrm{E}-005$ & $2.2716 \mathrm{E}-006$ & $1.4288 \mathrm{E}-007$ & $8.9515 \mathrm{E}-009$ & $5.5984 \mathrm{E}-010$ \\
\hline $1 / 128$ & $1.1588 \mathrm{E}-004$ & $8.0642 \mathrm{E}-006$ & $5.1092 \mathrm{E}-007$ & $3.2072 \mathrm{E}-008$ & $2.0072 \mathrm{E}-009$ \\
\hline \multicolumn{7}{|c|}{ Rashidinia et al. (2007) } & & & \\
\hline $1 / 16$ & $4.07 \mathrm{E}-005$ & $2.53 \mathrm{E}-006$ & $1.58 \mathrm{E}-007$ & $9.87 \mathrm{E}-009$ & $6.17 \mathrm{E}-010$ \\
\hline $1 / 32$ & $2.00 \mathrm{E}-005$ & $1.24 \mathrm{E}-006$ & $7.74 \mathrm{E}-008$ & $4.83 \mathrm{E}-009$ & $3.02 \mathrm{E}-010$ \\
\hline $1 / 64$ & $5.45 \mathrm{E}-005$ & $3.42 \mathrm{E}-006$ & $2.14 \mathrm{E}-007$ & $1.34 \mathrm{E}-008$ & $8.39 \mathrm{E}-010$ \\
\hline $1 / 128$ & $1.83 \mathrm{E}-004$ & $1.22 \mathrm{E}-005$ & $7.68 \mathrm{E}-007$ & $4.81 \mathrm{E}-008$ & $3.01 \mathrm{E}-009$ \\
\hline Kadalbajoo and Bawa $(1996)$ & & & \\
\hline $1 / 16$ & $7.09 \mathrm{E}-003$ & $1.77 \mathrm{E}-003$ & $4.45 \mathrm{E}-004$ & $1.11 \mathrm{E}-004$ & $2.78 \mathrm{E}-005$ \\
\hline $1 / 32$ & $5.68 \mathrm{E}-003$ & $1.42 \mathrm{E}-003$ & $3.55 \mathrm{E}-004$ & $8.89 \mathrm{E}-005$ & $2.22 \mathrm{E}-005$ \\
\hline $1 / 64$ & $4.07 \mathrm{E}-003$ & $1.01 \mathrm{E}-003$ & $2.54 \mathrm{E}-004$ & $6.35 \mathrm{E}-005$ & $1.28 \mathrm{E}-005$ \\
\hline $1 / 128$ & $6.97 \mathrm{E}-003$ & $1.75 \mathrm{E}-003$ & $4.33 \mathrm{E}-004$ & $1.08 \mathrm{E}-004$ & $2.71 \mathrm{E}-005$ \\
\hline Surla et al. $(1991)$ & & & & \\
\hline $1 / 16$ & $4.14 \mathrm{E}-003$ & $1.02 \mathrm{E}-003$ & $2.54 \mathrm{E}-004$ & $6.35 \mathrm{E}-005$ & $1.58 \mathrm{E}-005$ \\
\hline $1 / 32$ & $3.68 \mathrm{E}-003$ & $9.03 \mathrm{E}-004$ & $5.61 \mathrm{E}-005$ & $1.40 \mathrm{E}-005$ & $3.50 \mathrm{E}-006$ \\
\hline $1 / 64$ & $3.45 \mathrm{E}-003$ & $8.40 \mathrm{E}-004$ & $2.08 \mathrm{E}-004$ & $5.20 \mathrm{E}-005$ & $1.30 \mathrm{E}-005$ \\
\hline $1 / 128$ & $3.43 \mathrm{E}-003$ & $8.21 \mathrm{E}-004$ & $2.03 \mathrm{E}-004$ & $5.06 \mathrm{E}-005$ & $1.26 \mathrm{E}-005$ \\
\hline
\end{tabular}


The computational rate of convergence can also be obtained by using the double mesh principle defined below.

Let $\quad Z_{h}=\max _{i}\left|y_{i}^{h}-y_{i}^{h / 2}\right|, \quad i=1,2, \ldots, N-1$

Where, $y_{i}^{h}$ is the numerical solution on the mesh $\left\{x_{i}\right\}_{1}^{N-1}$ at the nodal point $x_{i}$ where $x_{i}=x_{0}+i h, \quad i=1,2, \ldots, N-1$ and where, $y_{i}^{h / 2}$ is the numerical solution at the nodal point $x_{i}$ on the mesh $\left\{x_{i}\right\}_{1}^{2 N-1}$ where, $x_{i}=x_{0}+i h / 2, \quad i=1,2, \ldots, 2 N-1$.

In the same way one can define $Z_{h / 2}$ by replacing $h$ by $h / 2$ and $N-1$ by $2 N-1$.

That is, $z_{h / 2}=\max _{i}\left|y_{i}^{h / 2}-y_{i}^{h / 4}\right|, i=1,2,3, \ldots, 2 N-1$.

The computed rate of convergence is defined as

$$
\text { Rate }=\frac{\log Z_{h}-\log Z_{h / 2}}{\log 2} .
$$

Tables 3 and 4 shows the rate of convergence of the present method for different values of the mesh size $h$.

Table 3. Rate of Convergence for Example $1(\varepsilon=1 / 16)$

\begin{tabular}{|cccccc|}
\hline$h$ & $h / 2$ & $Z_{h}$ & $h / 4$ & $Z_{h / 2}$ & Rate \\
\hline $1 / 16$ & $1 / 32$ & $1.8371 \mathrm{E}-006$ & $1 / 64$ & $1.1566 \mathrm{E}-007$ & 3.9894 \\
\hline $1 / 32$ & $1 / 64$ & $1.1566 \mathrm{E}-007$ & $1 / 128$ & $7.2420 \mathrm{E}-009$ & 3.9974 \\
\hline $1 / 64$ & $1 / 128$ & $7.2420 \mathrm{E}-009$ & $1 / 256$ & $4.5302 \mathrm{E}-010$ & 3.9987 \\
\hline
\end{tabular}

Table 4. Rate of Convergence for Example $2(\varepsilon=1 / 16)$.

\begin{tabular}{|cccccc|}
\hline$h$ & $h / 2$ & $Z_{h}$ & $h / 4$ & $Z_{h / 2}$ & Rate \\
\hline $1 / 16$ & $1 / 32$ & $2.5453 \mathrm{E}-005$ & $1 / 64$ & $1.5830 \mathrm{E}-006$ & 4.0071 \\
\hline $1 / 32$ & $1 / 64$ & $1.5830 \mathrm{E}-006$ & $1 / 128$ & $9.8814 \mathrm{E}-008$ & 4.0018 \\
\hline $1 / 64$ & $1 / 128$ & $9.8814 \mathrm{E}-008$ & $1 / 256$ & $6.1738 \mathrm{E}-009$ & 4.0005 \\
\hline
\end{tabular}

Figures 1(a) - 2(b) show that the numerical solutions obtained by the present method for $h \geq \varepsilon$ as compared to the exact solutions. 


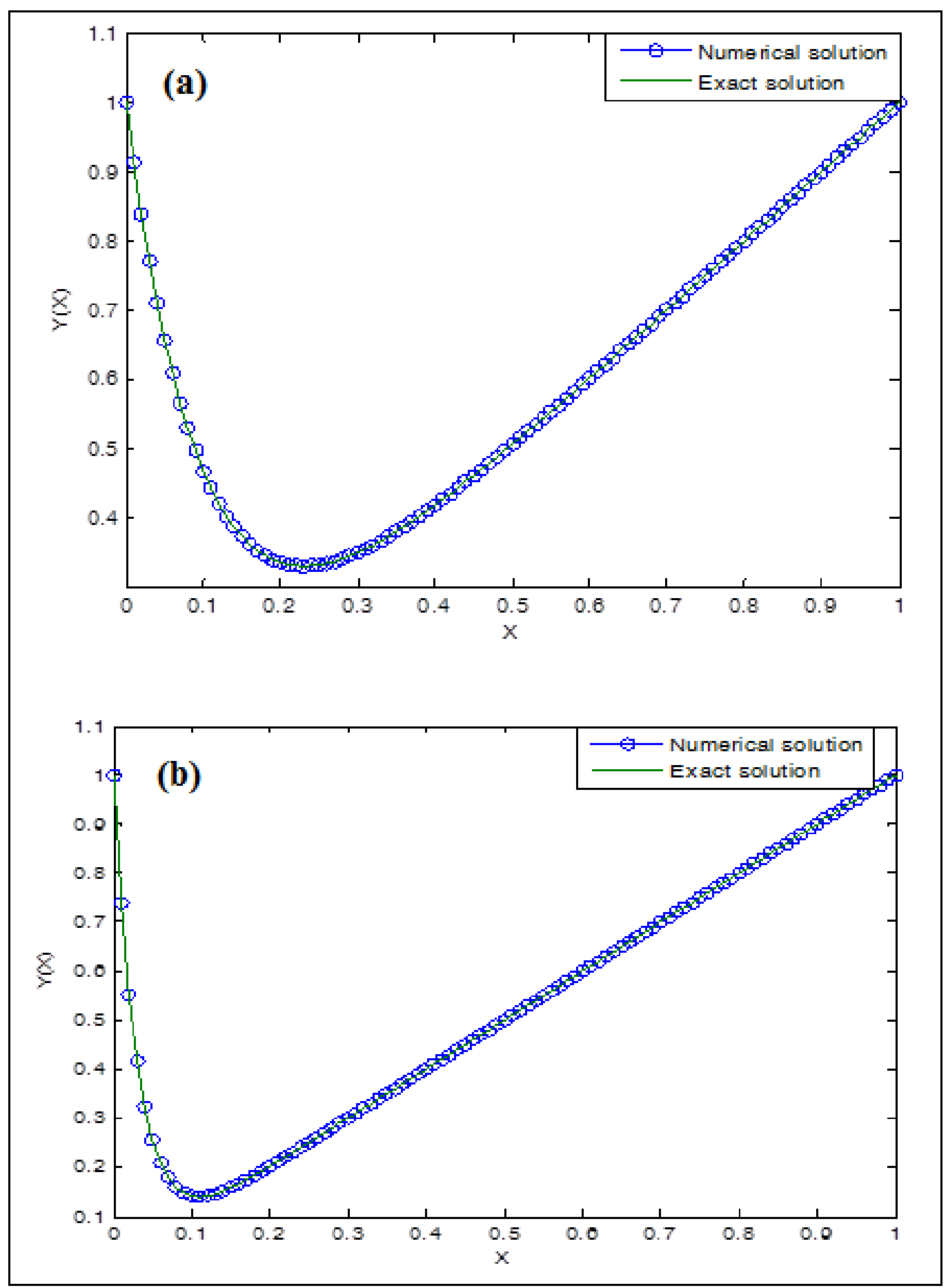

Figure 1. (a) Numerical Solution of Example 1 for $\varepsilon=0.01 \& h=0.01$., (b) Numerical Solution of Example 1 for $\varepsilon=0.001 \& h=0.01$. 


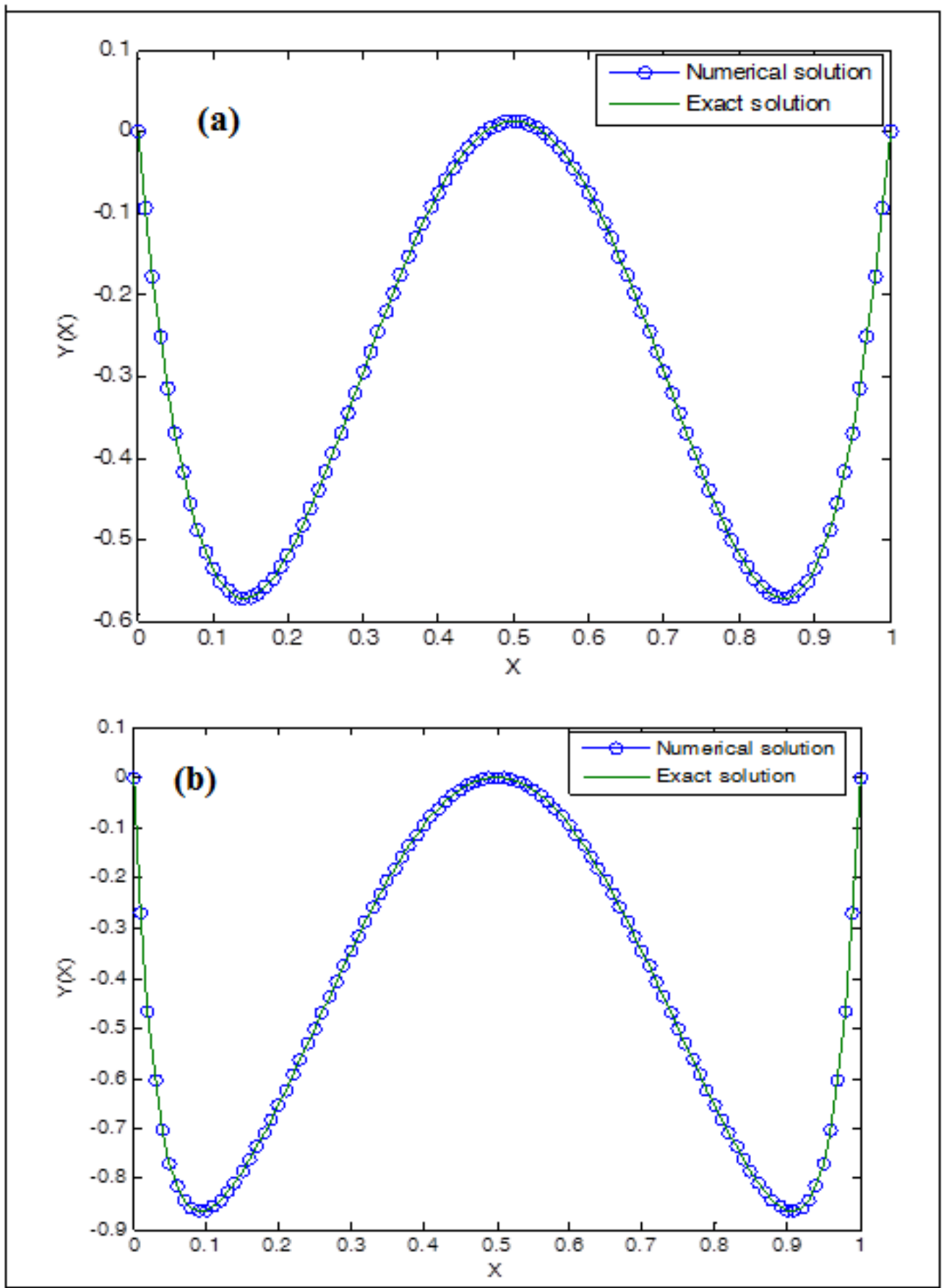

Figure 2. (a) Numerical solution of Example 2 for $\varepsilon=0.01 \& h=0.01$., (b) Numerical solution of Example 2 for $\varepsilon=0.001 \& h=0.01$. 


\section{DISCUSSION}

In this paper, fourth order compact finite difference method has been presented for solving singularly perturbed reaction-diffusion equations. To validated the applicability of the method, two model examples have been solved by taking different values for the perturbation parameter, $\varepsilon$ and mesh size, $h$. The numerical results obtained by the present method have been tabulated in terms of maximum absolute errors for different values of the perturbation parameter, $\varepsilon$ and mesh points, $N$; and compared with numerical results obtained by (Rashidinia et al., 2007; Kadalbajoo and Bawa, 1996; Surla et al., 1991). Further, the numerical solutions obtained by the proposed method for $h \geq \varepsilon$, for which most of the existing numerical methods fail to give good results, have been presented in graphs (Figures $1(\mathrm{a} \& \mathrm{~b})$ and $2(\mathrm{a} \& \mathrm{~b})$. As it can be observed from the tables and graphs, the present method approximates the exact solution very well and gives better solution than some existing methods reported in the literature.

Both the theoretical and numerical order of convergence have been investigated (Section 3; and Tables $3 \& 4$ ) and the results obtained confirmed that computational rate of convergence is in agreement with the theoretical estimates of the order convergence. In concise manner, the present method is conceptually simple, easy to use and readily adaptable for computer implementation for solving singularly perturbed reaction-diffusion equation.

\section{CONCLUSION}

Fourth order compact finite difference method has been presented for solving singularly perturbed reaction-diffusion equations. The method approximates the exact solution very well and gives better result than some existing methods reported in the literature. The rate of convergence of this method has been computed and is observed that it is in agreement with the theoretical estimates of the method which is of fourth order convergent.

\section{ACKNOWLEDGMENTS}

The authors would like to thank Jimma University for the financial and materials support as the work is the part of the MSc Thesis of Mr. Fasika Wondimu which is supported by the university. 


\section{REFERENCE}

Angel E \& Bellman, R. 1972. Dynamic programming and partial differential equations. ISBN: 978-94-009-5209-6, Academic Press, New York, pp.28-35.

Bellman, R. 1964. Perturbation techniques in mathematics, physics and engineering. ISBN: 0486-43258-0, Holt, Rinehart \& Winston, New York, pp.20-31.

Bender, C.M \& Orszag, S.A. 1978. Advanced mathematical methods for scientists and engineers. ISBN: 978-1-4757-3069-2, McGraw-Hill, New York, pp.419-575.

Cole, J.D \& Kevorkian, J. 1979. Perturbation methods in applied mathematics. ISBN: 00665452, .Springer, New York, pp.481-545,

Doolan, E.P., Miller, J.J.H \& Schilders, W.H.A. 1980. Uniform numerical methods for problems with initial and boundary layers. Boole Press, Dublin, 524p, DOI:10.1002/nme.1620180814.

Eckhaus, W. 1973. Matched asymptotic expansions and singular perturbations, Elsevier, ISBN: 008071178, 9780080871172, North-Holland, Amsterdam, 144p.

Elsgolt's, L. E \& Norkin, S. B. 1973. Introduction to the theory and applications of differential equations with deviating arguments. ISBN: 0080956149, 9780080956145, Academic Press, New York, 356p.

Geng, F. 2011. A novel method for solving a class of singularly perturbed boundary value problems based on reproducing kernel method, Applied Mathematics and Computation, 218(8): 4211-4215.

Goering, H., Felgenhauer, A., Lube, G., Roos, H.G \& Tobiska, L. 1983. Singularly perturbed differential equations. Lecture notes SS 2008, Academic Verlag, Berlin, 54p.

Hemker, P.W. 1977. A numerical study of stiff twopoint boundary problems. MCT 80, Mathematical Centre, ISBN: 9061961467, 9789061961468, Amsterdam, 178p.

Hemker, P.W \& Miller, J.J.H. (Eds.). 1979. Numerical analysis of singular perturbation problems. Academic Press, New York, 217-249 Description: xi, 499p.

Kadalbajoo, M. K \& Bawa, R. K. 1996. Variable-mesh difference scheme for singularly perturbed boundary value problems using Splines. Journal of Optimization Theory and Applications, 90: 405-416.

Miller, J.J.H. (Ed.). 1993. Application of advanced computational methods for boundary and interior layers. ISBN-13: 978-1857480023, Boole Press, Dublin, 220p.

CNCS, Mekelle University

ISSN: 2220-184X 
Miller, J.J.H., O’Riordan, E \& Shishkin, G.I. 1996. Fitted Numerical Methods for Singular Perturbation Problems: Error estimates in the maximum norm for linear problems in one and two dimensions. ISBN: 978-981-02-2462-2, World Scientific, River Edge, NJ07661, USA, 180p.

Mohanty, R. K \& Jha, N. 2005. A class of variable meshes spline in compression methods forsingularly perturbed two-point singular boundary-value problems. Applied Mathematics and Computation, 168: 704-716.

Nayfeh, A.H. 1973. Perturbation methods. ISBN: 0-521-37310-7, Wiley, New York, 4p.

Nayfeh, A.H. 1981. Introduction to perturbation techniques. ISBN: 978-1-4614-5476-2, Wiley, New York.

O'Malley, R.E. 1974. Introduction to singular perturbations. ISSN: 0036-1445, Academic Press, New York.

O'Malley, R.E. 1991. Singular perturbation methods for ordinary differential equations. ISBN: 978-1-4612-6968-7, Springer, New York, pp. 22-200.

Padmaja, P., Chakravarthy, P. P \& Reddy, Y. N. 2012. A nonstandard explicit method for solving singularly perturbed two point boundary value problems via method of reduction of order, International Journal of Applied Mathematics and Mechanics, 8(2): 62-76.

Prasad, H. Sh \& Reddy, Y. N. 2011. Numerical treatment of singularly perturbed two-point boundary value problems with mixed condition using differential quadrature method. International Journal of Applied Science and Engineering, 9(3): 207-221.

Rashidinia, J., Ghasemi, M \& Mahmoodi, Z. 2007. Spline approach to the solution of singularlyperturbed boundary-value problems. Applied Mathematics and Computation, 189: 72-78.

Surla, K., Herceg, D \& Cvetkovic, L. 1991. A family of exponential spline difference scheme. Review of Research Faculty of Science Mathematics Series, 19: 12-23.

Van Dyke, M. 1975. Perturbation methods in fluid mechanics. Parabolic Press, Stanford, CA. pp.149-163. 